\title{
Ultrasonic time reversal for haptic interaction
}

\author{
Dipl.-Ing. Sebastian Wöckel, Dipl.-Ing. Hendrik Arndt, Dr.-Ing. Ulrike Steinmann, \\ B.Sc. Benjamin Bedürftig, Prof. Dr. Jörg Auge \\ Institut für Automation und Kommunikation (ifak) e.V. Magdeburg, Department Measurement \\ Technology and Power Electronics, Werner-Heisenberg-Str. 1, 39106 Magdeburg, Germany \\ sebastian.woeckel@ifak.eu
}

\begin{abstract}
:
The reversibility of an acoustic wave can be used to refocus a signal on a point by time reversal (TR) techniques. Numerous application fields are connected to this technology, such as medical intervention, non-destructive testing or impact localization on touchscreen systems. Latter technology recently gains increasing attention. Normally touchscreens require the users to visually control their interaction. However, if this is not possible, e.g. in cases of individual physical constraints or environmental conditions, the usability is limited due to a missing alternative feedback. A lot of simple localization approaches exists using the acoustic interaction of a finger impact. But the reverse local haptic feedback with elastic waves is not sufficiently supported up to now. Here, TR can provide a user-oriented support by realizing vibro-tactile sensations at virtual buttons or other control elements on the screen. Therefore the contribution addresses the local haptic feedback on panels by using a limited number of acoustic transducers, supported by simulation-based studies.
\end{abstract}

Key words: haptic interaction, time reversal, structure borne sound, numerical calibration

\section{Motivation}

Operating a touch-screen, as in case of a smartphone, tablet, GPS device or public cashmachine, meanwhile is a matter of course in our everyday life (Fig. 1). However, using a touchsensitive display is not taken for granted for everyone (e.g. in case of individual physical constraints) or in everyday situation (e.g. unfavorable external conditions). Then, the usability is limited due to an absent haptic feedback as in case of real keys or buttons.

\section{According scenarios are:}

- safety-related situations where particular care has to be taken to avoid distractions or loss of attention, e.g. operating the GPS device during driving,

- technical constraints, e.g. blinding by sunlight, poor visibility of the display,

- physical constraints, e.g. ability to see,

- situational context, e.g. during sports.

The controllability of such touch-sensitive devices must succeed also under those conditions. A prospective user-oriented support for instance addresses pushing a button or other interaction with the screen.

For haptic feedback the authors want to utilize ultrasonic waves in terms of a local vibro-tactile sensation (impulse, vibration) at one or several positions on the screen. Due to a local interference of acoustic waves a sensible mechanical signal arises. Thereby, visually accessible elements can be supported by haptic feedback.

The technique behind it bases on the acoustic time reversal. Time Reversal (TR) in acoustics is subject of different international research activities, starting with the work of Fink et al. (c.f. [1], [2]) in the early 1990's. The approach takes advantage of the reversibility of acoustic

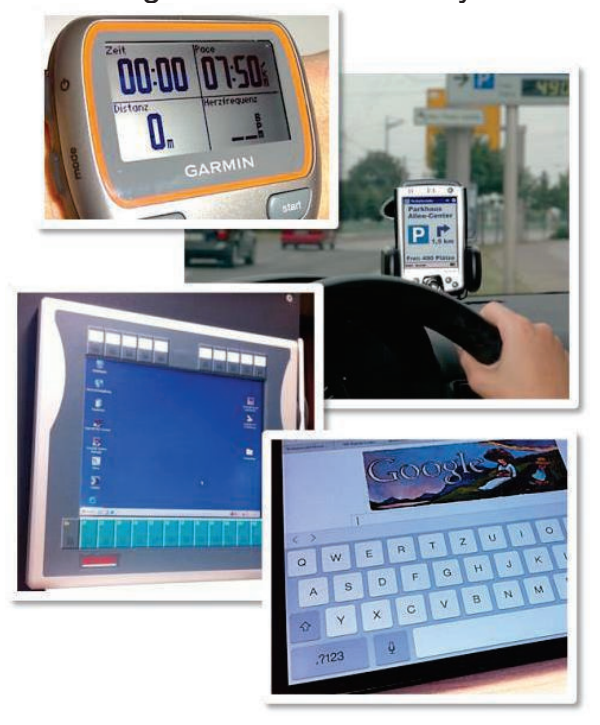

Fig. 1. Exemplary applications for haptic feedback. 


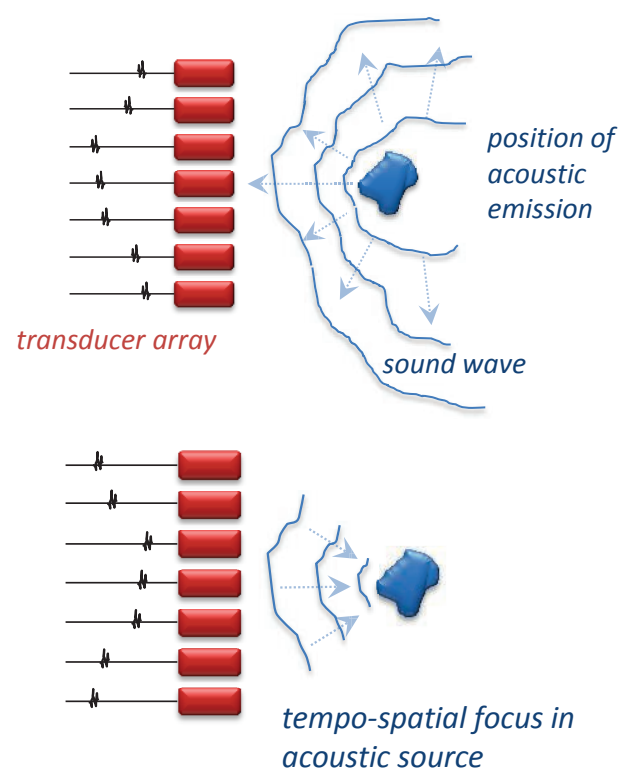

Fig. 2. Scheme of acoustic time reversal: (1) acoustic emission within a test medium and receiving the signal with a transducer array, (2) time-reversed transmission, back-propagation and constructive interference at the acoustic origin.

wave propagation in a non-dissipative medium, where signals are back-propagated to the sources that emitted them (Fig. 2). Numerous application fields are connected to this technology, such as underwater communications, medical imaging and intervention or non-destructive testing.

An aim of current R\&D activities is to establish haptic feedback on panels and planar structures by using a limited number of acoustic transducers. In contrast to known calibration procedures no experimental training stage to set up a space-resolved database of plate impulse responses is necessary. Instead, the transient signal at the transducer array, that is required to selectively address a point of feedback on the panel, is gained from numerical simulations. Moreover, simulation is used to define

- the actuators signals with respect to the contrast and resolution of tactile sensation in a focal point,

- the potential to selectively focus on multiple points (Fig. 3 a) at the same time and through an intermediate layer (Fig 3 b) and

- the main components of the wave propagation paths to reduce the excitation length of the time reversed signal (Fig. 4) improving a fast consecutive haptic feedback.

Additionally, the influence of focal point distance to the actuators and thus the decrease of the out-of-plane displacement are investigated. Numerical results are evaluated by measurements and will be discussed.

Another objective of the contribution is to analyze appropriate strategies to influence the signal content. On the one hand this concerns mode selectivity in order to excite mainly (fundamental) Lamb wave modes. For this, suitable transducers and filter elements are tested. On the other hand the necessary duration of the actuator signals (time-scale windowing) as well as the consistency of simulated and experimental excitation signals and the respective impacts on the focal quality are evaluated.

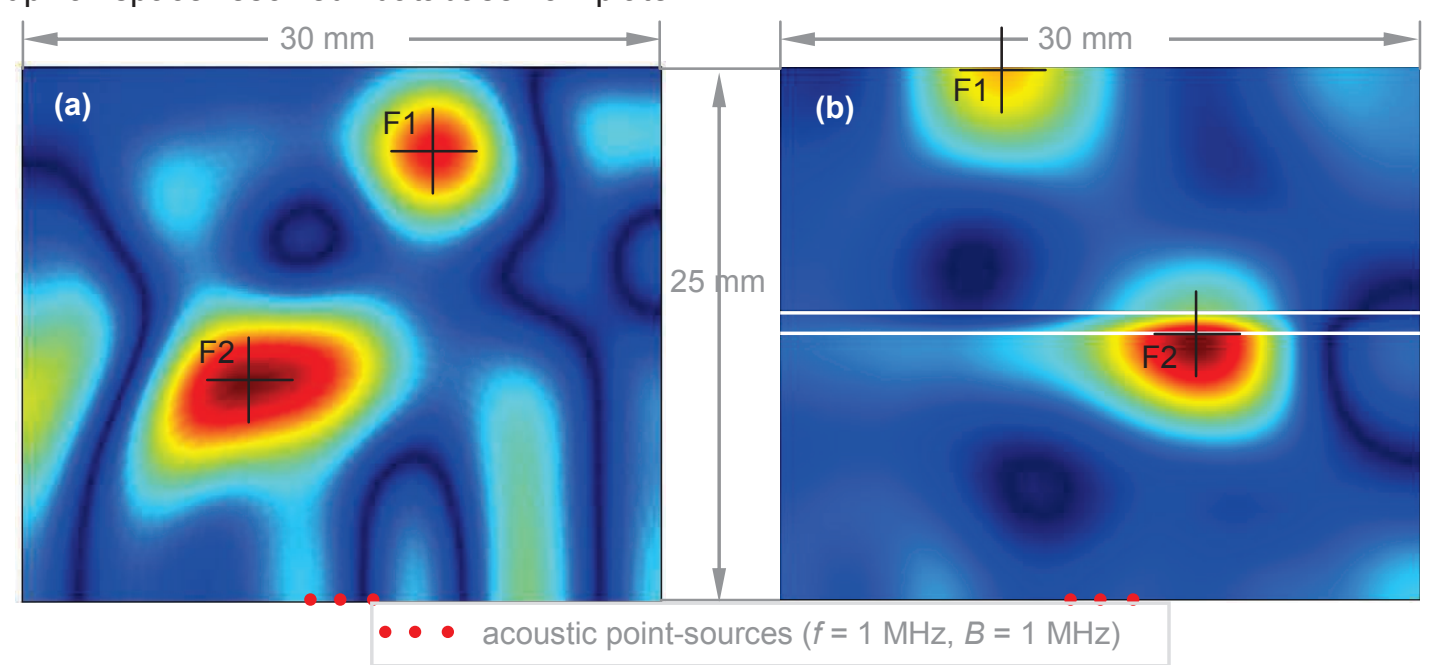

Fig. 3: FE-Simulation of the absolute pressure distribution when focusing on two different points F1 and F2 within the focal beam width at the same time with direct time reversal using 3 point sources at center frequency of $f=1$ $\mathrm{MHz}$ and bandwidth of $B=1 \mathrm{MHz}$. (a) Homogeneous medium with sound-hard boundaries. (b) Inclusion of an intermediate layer with higher acoustic impedance. The intended focal point positions are different to (a) and directly placed at an interface and the boundary. 

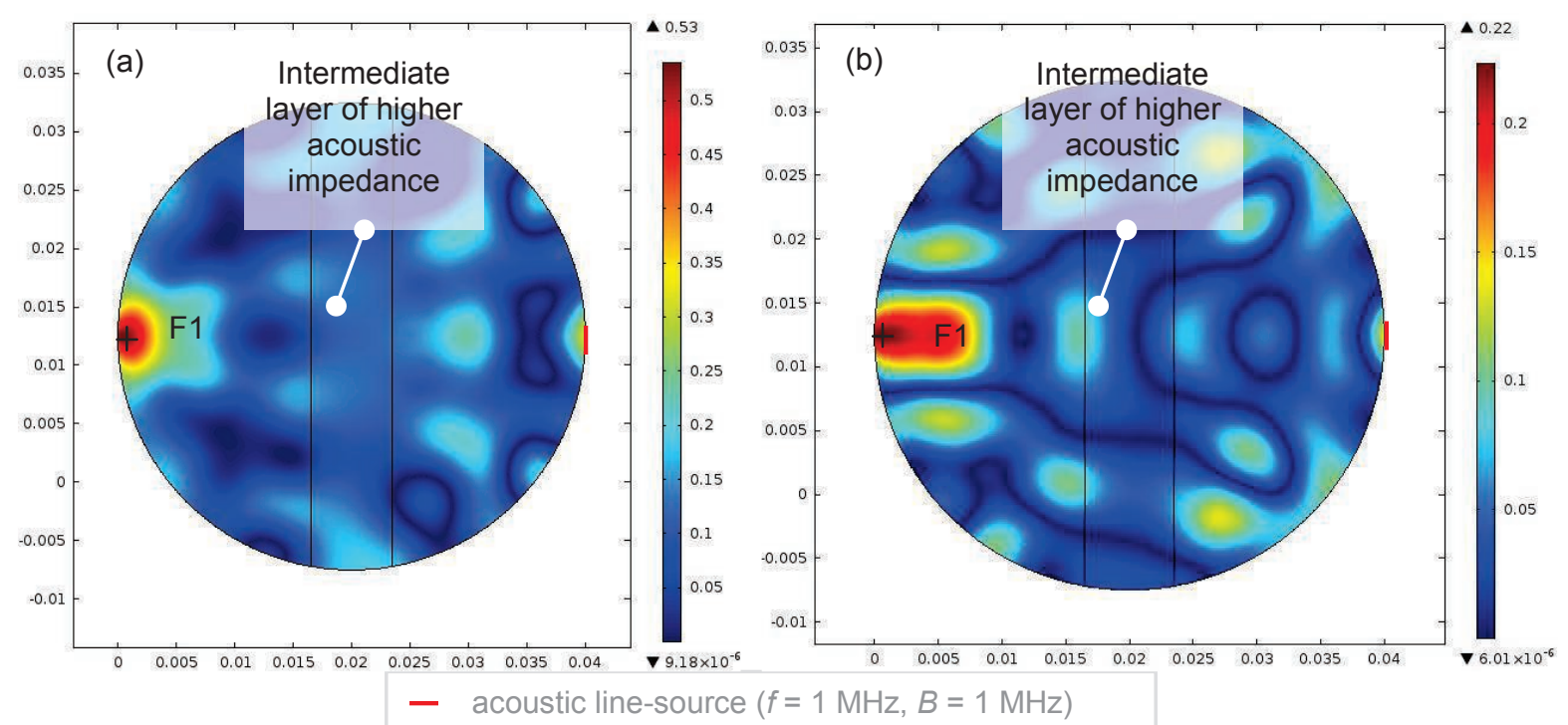

Fig. 4: FE-Simulation of the absolute pressure distribution (normalized on excitation amplitude): (a) Focusing through an intermediate layer of higher acoustic impedance on a single focal point F1. (b) Focusing on F1 with a reduced time reversed signal to a few main components (30\% of original signal length used in (a)). A widening of the focal point and a reduction of the signal-to-clutter ratio related to the focus amplitude and the secondary clutter amplitudes is observed.

\section{Basic physic conditions for haptic feedback}

The haptic feedback that can be felt by a human fingertip (haptic perceptual detection threshold) depends on the vertical and horizontal displacement of the surface (acoustic energy), the excitation frequency (wavelength) and the shape of the transient displacement. The maximum of the haptic sensitivity can be found at a vibration frequency of $f=200 \ldots 300 \mathrm{~Hz}$. The corresponding sensitivity levels (perceptual threshold) of the elongation at these frequencies are in the range of $\Delta x>0.2 \mu \mathrm{m}$ for transversal displacement and $\Delta z>5 \mu \mathrm{m}$ for displacements of the surface vertical to the skin surface. A cumulative movement of the finger over the surface additionally lowers the sensitivity level.

Due to the ratio of the propagation velocity (larger wavelength) to the dimension of the focus point the shear mode (e.g. Rayleigh shear horizontal $\mathrm{SH}$ ) is unsuitable despite the higher sensitivity $(0.2 \mu \mathrm{m})$. Accordingly the presented work concentrates on the basic asymmetric mode $\mathrm{A} 0$. The usage of plate related eigenmodes further improve the vertical elongation.

A stimulation signal in the range of the perceptual detection maximum $(200 \ldots 300 \mathrm{~Hz})$ will cause minor technical efforts because only low electric power is needed. The spatial resolution depends on the wavelength. Signals with higher frequencies will need a higher excitation power due to the lower surface displacement in this frequency range. Furthermore, additional acoustic perceptible disturbing signals would occur, which normally are not wanted.

Typically common touch sensitive displays, which are in need of haptic feedback, are made of glass or plastic material with a thickness in the range of $2 \mathrm{~mm}$ to $5 \mathrm{~mm}$ [3]. Because of the larger physical wavelength on such plates neither local steplike thresholds nor multiple arbitrary focus points in size and spacing of a human fingertip can be realized at the haptic frequencies $(<300 \mathrm{~Hz})$. Thus, the selective generation of larger feedback spots on comparatively large plate structures seems to be realistic for now.

\section{D-FE-Model}

In theory there is an unlimited number of different guided modes of structural elastic waves that can propagate on plate structures. The phase and group velocities of each mode are dispersive and a function of frequency. Hence, it is necessary to evaluate each mode concerning its cut-off frequency, wavelength and the vertical displacement, which is supposed to be the main parameter for haptic interaction. For solving the differential equations that describe the elastic transient behavior depending on the geometry (plate thickness), the elasticity and the Poisson number a numeric two dimensional model is used. The model consists of a plate slice assuming Floquet periodicity of the displacement vector. 


\section{Dispersion}

The solution of the phase velocities as a function of frequency based on the finite elements simulation enables the geometric design of the plate and the selection of single wave modes towards an optimized haptic feedback. Normally the solution is depicted within a dispersion diagram. As an example Fig. 5 represents the dispersive characteristics of the velocities for an acrylic material and Fig. 6 depicts the wavelengths for two typical materials used for displays in comparison to a steel plate. Table 1 summarizes the corresponding acoustic parameters. In the range of the haptic frequency $(300 \mathrm{~Hz})$ the wavelength varies from $160 \mathrm{~mm}$ to $290 \mathrm{~mm}$ for the asymmetric mode $\mathrm{A} 0$. The next higher Rayleigh-mode $\mathrm{SHO}$ has a quite larger wavelength (Fig. 5), which is not suitable for high spatial resolution in focal points.

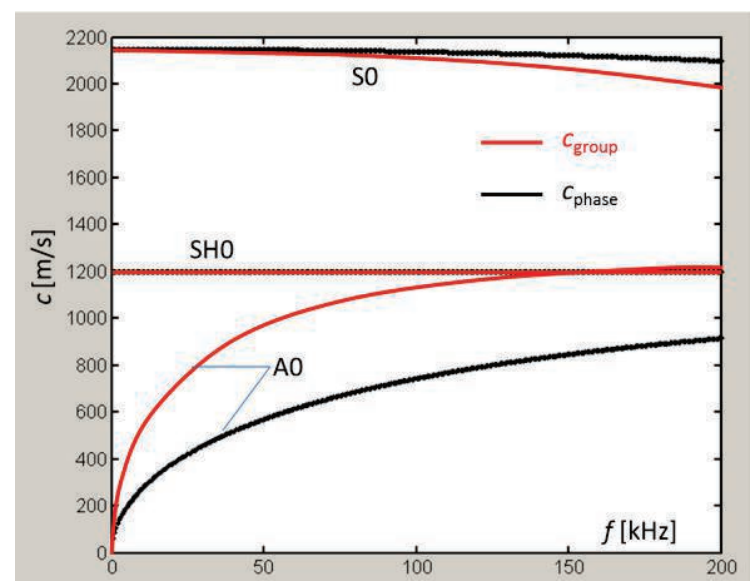

Fig. 5. Dispersion diagram for phase and group velocities of asymmetric (AO), symmetric (SO) lamb modes and Rayleigh mode (SHO) on an acrylic plate with thickness of $d=2 \mathrm{~mm}$.

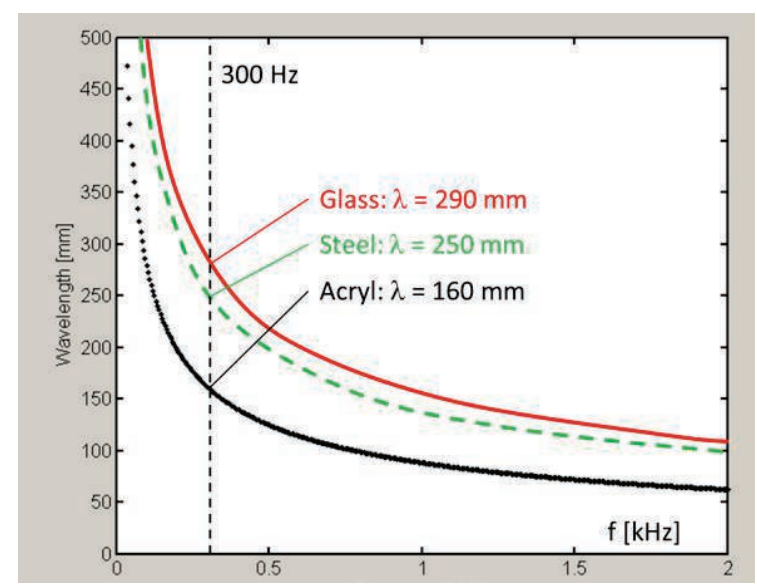

Fig. 6. Wavelength of asymmetric lamb-mode $A O$ for plates with thickness of $d=2 \mathrm{~mm}$ and different materials (refer to Tab.1).
Tab. 1: Material parameters

\begin{tabular}{|l|c|c|c|c|c|}
\hline Material & $\begin{array}{c}\rho \\
{\left[\mathrm{kg} / \mathrm{m}^{3}\right]}\end{array}$ & $\begin{array}{l}\text { CLong. } \\
{[\mathrm{m} / \mathrm{s}]}\end{array}$ & $\begin{array}{l}\text { CTrans. } \\
{[\mathrm{m} / \mathrm{s}]}\end{array}$ & $\begin{array}{c}\mathrm{E} \\
{[\mathrm{GPa}]}\end{array}$ & $\mu$ \\
\hline Acryl & 1200 & 2700 & 1200 & 4,76 & 0,38 \\
\hline Glass & 1590 & 6790 & 4321 & 68.9 & 0.16 \\
\hline Steel & 7850 & 5778 & 3194 & 205 & 0,28 \\
\hline
\end{tabular}

\section{D-FE-Model}

A three dimensional model for plates was created to solve for the stationary eigenmodes and the transient behavior of short time pulses that are excited from different multiple transducer locations (Fig. 7). Especially the reflection and mode conversion at the boundaries needs to be modelled because it is essential for the time reversal process with a reduced number of excitation points. The parameter studies covered variations of the number and placement of the source points, the plates' material and thickness, the frequency and fixed and floating bearing of the boundaries. For the sake of briefness only the influence of source patterns is discussed in this contribution.

One main goal of the simulation is to provide a virtual description of the necessary input data at the transducers in order to excite a time reversed signal at a desired focus location at the empirical experimental verification step.

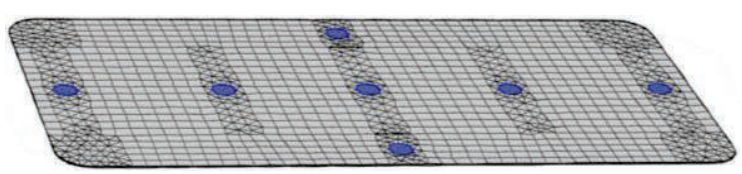

Fig. 7. 3D-FE-Model of a plastic plate $(1 \mathrm{~m} \times 0,5 \mathrm{mx}$ $2 \mathrm{~mm}$ ) with discretization for $f=300 \mathrm{~Hz}$. The excitation points are marked by blue circles.

As an example the simulation results for different plastic and glass plates are illustrated in Fig. 8, 9 and 10. Fig. 8 and 9 depict the forward-propagation of an elastic impulse at the desired focal point in case of varying material and plate dimension. The ideal replication of the original impulse by time reversal of the divergent wave field requires a time reversal mirror, whose unlimited number of excitation points forms a curve integral surrounding the original excitation point and gathering the total transient acoustic field crossing that curve. Alternatively, an unlimited number of multiple reflections (without damping) is needed to approximate the same divergent wave field by virtual sources at the reflecting boundaries. Normally damping will occur and only a limited number of excitation points will be applicable. 
Hence, their number and positions need to be adapted to approximate the original signal. In the current study a reduced number of up to 16

$5 \mathrm{~ms}$

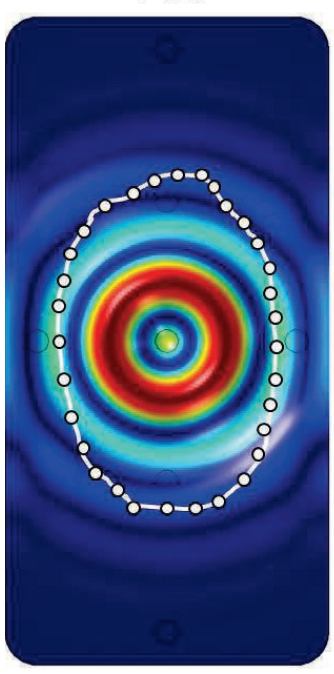

$7 \mathrm{~ms}$

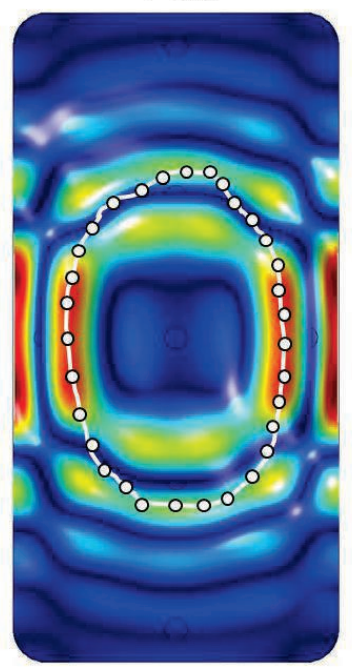

transducers with regular and randomized patterns were used. The plate's size was limited to $1 \mathrm{~m}^{2}$.

$9 \mathrm{~ms}$

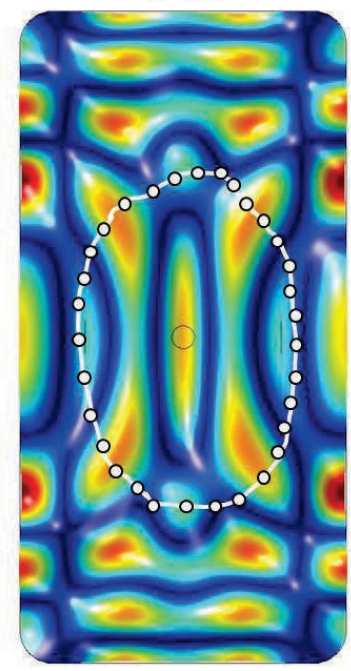

$11 \mathrm{~ms}$

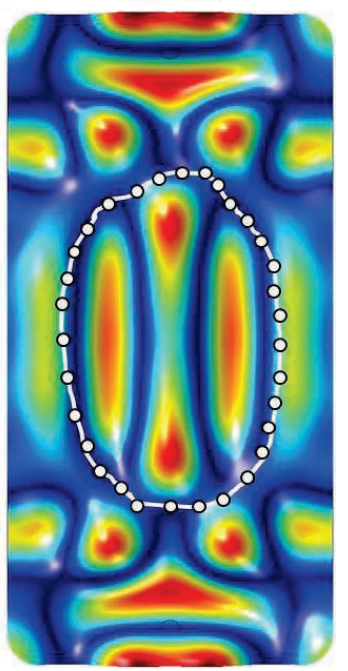

Fig. 8: Displacement on an acrylic plate $(1 \mathrm{~m} \times 0.5 \mathrm{~m} \times 2 \mathrm{~mm})$ caused by a gaussian excitation of an A0-wave with $f=300 \mathrm{~Hz}\left(c_{A O}=48 \mathrm{~m} / \mathrm{s}, \lambda=160 \mathrm{~mm}\right)$ in the middle of the plate. The max. elongation is about $\Delta z=+30 \mu \mathrm{m}$. The white curve represents an ideal time reversal mirror surrounding the focal point in the centre.

The 3D-solution of the transient divergent wave fields generated by different excitation (and later focus) points (Fig. 8 and 9) helps to determine the needed positions and number of source points for time reversal.
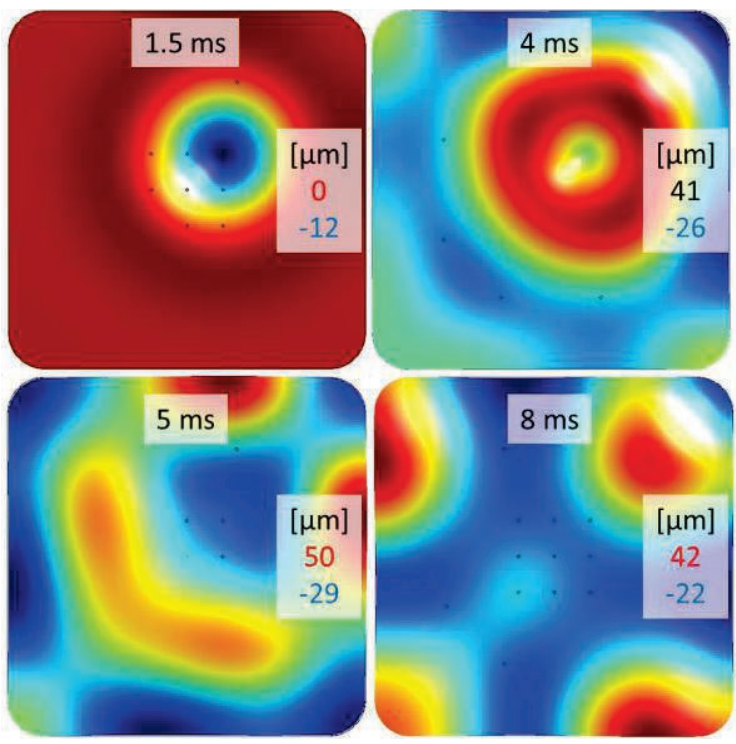

Fig. 9. Forward-Propagation

(vertical displacement) of a gauss pulse (asymmetric lambmode $A 0 C_{A O}=87 \mathrm{~m} / \mathrm{s}, \lambda=290 \mathrm{~mm}$ ) on a glass plate [500 $\times 500 \times 2] \mathrm{mm}$ for different time steps.

Based on the wave field snapshots of two chosen examples it can be seen that a regular pattern with a large number of transducers would give best results for focusing in the plate center (Fig. 8). In contrast a shifted, off-center focus requires an irregular pattern. In Fig. 9 the time steps $t=1.5 \mathrm{~ms}$ and $t=8 \mathrm{~ms}$ represent the ideal focal time for haptic feedback and the ideal source pattern for a time reversal.

A symmetric placement of the transducer will force a symmetric wave pattern on the plate which could mask a focus displacement. Hence, to avoid predominant wave patterns, a randomized transducer placement is necessary. Fig. 10 illustrates the focus quality by time reversal for a regular symmetric transducer pattern and a randomized (not optimized) pattern with 8 source points. In the left figure the interference with the predominant symmetric wave pattern lead to an insufficient replication of the focal point (white circle). In contrast the irregular transducer arrangement (right figure) supports the formation of a shifted focal point. Still, the focal point haptic discretization and vertical displacement is dependent on the physical wavelength.

Complementary to the numerical studies a measurement system was established which enables the time reversal investigation on plates with 16 independent channels. The elongation of the plate at arbitrary points is captured with a laser triangulation sensor. During the empirical studies a variety of electrodynamic and acoustic transducers (piezoceramic, vibration motor, structure-borne loudspeaker, voice coil motor) were tested towards their ability to excite an elastic AO wave on different plates in the haptic frequency range $(200 . .300) \mathrm{Hz}$. For the sake 
of briefness these results are not presented here, but will be discussed at the conference.

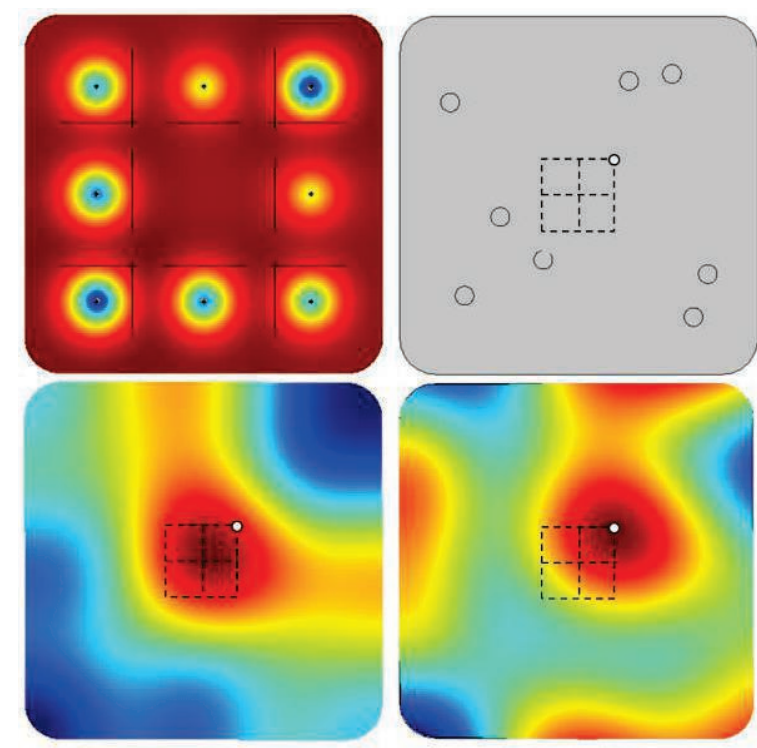

Fig. 10. Glass-plate $(500 \times 500 \times 2) \mathrm{mm}$ : Time reversal (simulation) with 8 transducers (excitation points) with regular (left) and randomized (right) pattern focusing on the same focal point (white spot) with a spatial offset to the plates center of $\Delta x=\Delta y=$ $+50 \mathrm{~mm}$ (wavelength at $325 \mathrm{~Hz}: \lambda_{\mathrm{AO}}=290 \mathrm{~mm}$ ): top: excitation timestep, down: focus timestep (time reversal)

\section{Summary}

The current studies illustrate that an arbitrary time and spatial focusing of an elastic impulse can be established in the perceptual haptic frequency range. The size of the focus is determined by the physical wavelength of approx. $10 \mathrm{~cm}$ on plastic or glass plates with a thickness of $2 \mathrm{~mm}$. Additionally, the haptic quality of the focal signal could be improved by switching the frequency to an eigenmode of the plate.

There is also a good agreement between the virtual calibration of the time reversal signals by simulations and the empirical results (not shown here). In further steps the combination of finger impact localization [7] and local haptic feedback will be investigated.

\section{Acknowledgement}

The project is supported by the German Federal Ministry of Education and Research (BMBF) under contract number 16SV7104.

\section{References}

[1] M. Fink, Time-Reversal of Ultrasonic Fields-Part I: Basic principle, IEEE Trans. Ultrason., Ferroelect., Freq. Contr. 39(5), 555-566 (1992); doi: 10.1109/58.156174

[2] N. Chackroun, M. Fink, F. Wu, Time-reversal processing in non-destructive testing, IEEE Trans. Ultrason. Ferroelec. Freq. Contr. 42 (6), 1087-1098 (1995); doi: 10.1109/58.476552

[3] Bai MR, Tsai YK., Impact localization combined with haptic feedback for touch panel applications based on the time-reversal approach. J Acoust Soc Am. 2011 Mar ;129(3):1297-305. doi: 10.1121/1.3533725.

[4] Ros Ki Ing, and Mathias Fink, Time reversal interactive objects, J. Acoust. Soc. Am. 115, 2589 (2004);

[5] HH King, R Donlin, B Hannaford, Perceptual thresholds for single vs. multi-finger haptic interaction - Haptics Symposium, 2010

[6] Pham, D.T., Al-Kutubi, M., Ji, Z., Yang, M., Wang, Z., Catheline, S.: Tangible acoustic interface approaches. In: Proceedings of IPROMS 2005 Virtual Conference, pp. 497-502. Elsevier, Amsterdam (2005)

[7] Ros Kiri Ing and Mathias Fink, In solid localization of finger impacts using acoustic time-reversal process, Appl. Phys. Lett. 87, 204104 (2005); 